\title{
Non-emergent Weekend ERCP: It Makes Sense-Now, Make It Work
}

\author{
John A. Martin ${ }^{1}$ \\ Published online: 21 May 2020 \\ (c) Springer Science+Business Media, LLC, part of Springer Nature 2020
}

Endoscopic retrograde cholangiopancreatography (ERCP) is one of the most complex and highest risk-endoscopic procedures [1]. ERCP requires a dedicated team possessing specific skills along with specialized endoscopic and radiologic equipment and devices. Not only does ERCP require a physician endoscopist specially trained to perform this procedure, but nursing staff and endoscopy technicians with specialized ERCP skills. In some localities, radiology technologists (RTs) must be present to operate the fluoroscopy equipment. Furthermore, a substantial proportion of ERCPs are performed with anesthesia support usually including a certified nurse anesthetist (CRNA) and a supervising anesthesiologist. Given all these resources-human and technological-it is no surprise that ERCP requires substantial time and effort to coordinate, stage, and execute, and is costly to undertake [2].

Fortunately, as with most medical procedures, elective or otherwise, the vast majority of ERCPs are performed during standard weekday work hours, when clinical support staff and resources are most available, and, hence, efficiencies and economies-of scale-exceed those that can be marshalled over a weekend. Nevertheless, ERCPs are occasionally bona fide medical emergencies, and must be performed afterhours or on weekends and holidays. Multiple studies have demonstrated that ERCP performed after-hours or over the weekend can be as safe and effective as those performed during standard weekday hours [3-5], despite the potential for comparatively reduced clinical and clerical support.

Increasingly, however, weekend ERCP services are requested for less-than-emergent—or even elective-indications, on hospital inpatients, in situations where delaying the ERCP until Monday would not negatively impact clinical outcome. And while the ERCP procedure itself is costly, so is each additional day an inpatient spends in the hospital,

John A. Martin

Martin.John3@mayo.edu

1 Division of Gastroenterology and Hepatology, Mayo Clinic, Rochester, MN 55905, USA explaining this trend as an effort to reduce the length of hospital stay (LOS), when considered from the individual and combined standpoints of the patient, the payer, and the hospital-each of whom is poised to benefit in one or more ways from decreased LOS resulting directly from nonemergent weekend ERCP (assuming that the patient is actually discharged earlier as a result of undergoing ERCP over the weekend instead of waiting until Monday). By reducing the overall cost of care, decreasing excess LOS can be an important driver of improved value in healthcare $[6,7]$. Reducing LOS decreases expenses for the patient and the payer, reduces costs for the hospital, and improves access to hospital beds for patients; the latter benefits the hospital financially and improves their ability to provide more expedient service for the next patient in need of care. Shortening LOS also reduces in-hospital adverse events [8] and can, in some instances, increase patient satisfaction $[9,10]$.

Conversely, though, there are two potential groups who may not share in those benefits: the clinical caregivers and cost centers that provide non-emergent weekend ERCP. The clinical caregivers who provide non-emergent weekend ERCP service may well not benefit from such service if it is simply added on top of full weekday service without adjusting for this added workload by additional monetary compensation and/or compensatory time off during the workweek. Weekend on-call work may also require childcare and other family support that may be more difficult to procure outside of existing workweek arrangements and established support networks. The personal and financial impact on other clinical departments that support the ERCP procedure, such as anesthesia and radiology, must also be considered. These departments and cost centers bear the financial burden of paying the comparatively higher weekend hourly wages of staff who are called in-often at overtime pay rates of time-and-a-half or double time, not to mention a minimum of 2-4 h per call-in episode regardless of actual procedure duration -or, for salaried individuals, possibly additional or special pay related to on-call hours or compensatory time awarded as payback for weekend hours. Since cases are not 'stacked' as they are during weekdays, and since staffing is 
minimal throughout the hospital, lower efficiency compared with weekdays also potentially contributes to increased time and expense per case. Also, in the DRG-driven model of inpatient care, while the hospital saves costs through reduced LOS through lower cost to provide care over a shorter admission but with no decrease in the DRG payment, the procedure-providing cost center may lose, because the opportunity to provide a revenue-generating outpatient ERCP procedure has now been aggregated into a DRG payment, an example of yet another potential loss to the ERCP cost center in the midst of a win for the hospital. Unless the hospital, which saves money through non-emergent weekend ERCP service-associated decreased LOS, is willing to share such proceeds with the weekend ERCP-providing departments and cost-centers, the hospital wins financially, but the ERCP-providing cost centers do not: they lose. And they could lose much more than just money. These departments and cost centers that provide non-emergent weekend ERCP also must deal with potential secondary fallout arising from their employees being increasingly engaged in non-emergent weekend procedure service, particularly if such weekend service is provided without any compensatory diminishment of weekday clinical responsibilities for their staff, lest they hire a larger number of staff to absorb the overall increase in patient-care hours. Providing non-emergent weekend ERCP service, depending on paradigm of service delivery, can require recruitment of a larger cadre of ERCP-skilled physicians, nurses, and technicians in order to provide the additional service days and hours, unless existing staff desire to, and are able to actually fulfill the additional hours required above a full weekday schedule durably, and without detriment to their performance during regular weekday work hours and to their overall wellbeing. Otherwise, staff satisfaction and team morale could decline, and possibly contribute to increased employee turnover, particularly at institutions where nurses and technicians are able to leave the endoscopy unit and move to other departments in the enterprise that have less rigorous schedules, and are able to make such moves between departments without loss of compensation, benefits, or seniority. Such a resulting exodus would escalate the need to increase recruitment and train replacements, and perhaps even do so continuously if the exodus becomes frequent and regular. The result would be an unenviable vicious cycle of repeated additional recruiting and training expense and time investment, particularly given that physicians with expert ERCP skills, nurses, and endoscopy technicians are neither plentiful nor easily recruited, and that it takes many weeks, at minimum, even at the most expert and high-volume ERCP centers, to train nurses and technicians and equip them with ERCP skills adequate to perform the procedure as independent ERCP assistants, particularly at a level high enough to perform on a weekend day, when more experienced allied health colleagues with well-seasoned ERCP capabilities may not be on site and available to coach or supervise them.

In this issue of Digestive Diseases and Sciences, Hakim et al. [11], present a well-performed retrospective analysis of over 500 patients who underwent weekend ERCP consultation for non-emergent indications, and subsequently underwent ERCP either over the weekend, or early in the week. Outcomes of the weekend and early week groups were compared. Though a publication in the prior decade studying a smaller cohort of patients demonstrated weekend inpatient ERCP to be associated with a reduction in patient LOS [12], the study by Hakim et al., with a substantially larger patient sample, establishes not only that non-emergent weekend ERCP significantly reduces LOS, but that it also reduces hospital costs and charges, all while also increasing net hospital margins without negatively impacting the quality of clinical care. It follows, then, that providing non-emergent, and even elective, weekend ERCP services is beneficial in multiple ways for patients, payers, and hospital alike. How exactly, then, can non-emergent weekend ERCP become routine? For this to occur, the hospital must share their financial benefit with the clinicians and cost centers that provide the service. Effecting such change may require other alterations of the weekend endoscopy unit and hospital operations, since a threshold of case volume may be necessary to cover fixed and variable costs adequately and to achieve efficiencies of scale great enough to generate a positive margin of a magnitude large enough to be able to share meaningfully with the cost centers and clinicians-enough to make their implied sacrifices worth the effort, whether those sacrifices are measured in terms of time, money, or qualitative factors such as time missed with family, friends or loved ones. This may require some creative changes, such as shifting enterprise ERCPs to higher-capacity locations for weekend procedures, or scheduling a limited number of elective ERCPs and other endoscopies on weekend days without negatively impacting weekday volumes. Hospital organizations must step up and accept the challenge to help construct a weekend ERCP paradigm that is economically advantageous not only for the hospital enterprise, but also for the departments employing the physicians, anesthetists, nurses, technicians, and technologists - which, de facto, often financially operate as siloed cost centers which will not see the financial benefit of reduced hospital LOS anywhere on their own balance sheets-to make it affordable to compensate their staff adequately for taking on added weekend work on top of a full week of weekday work, or to hire additional staff to take what is currently organized as a 5 day work week, and turn it into a 6- or 7-day work week. The latter is tricky, as ERCPskilled physicians are not minted in large numbers, and CRNAs, RNs, LPNs, RTs, and endoscopy technicians who are in constant short supply and may be lured away to other departments within and outside their own institutions that 
do not require them to provide weeknight or weekend call services, or demand mandatory overtime. Hospital organizations, which, based on the data presented in this paper, are poised to benefit financially from this paradigm, must be willing to "share the wealth" therein forthcoming with the departments and teams of caregivers who would provide this weekend ERCP care in order to make non-emergent weekend ERCP service possible, all in the interest of best care for the patient and improvement of access to care.

Hakim, et al., have provided valuable insight into the premise that providing non-emergent weekend ERCP service may well make sense: by reducing LOS, decreasing hospital costs, and increasing hospital margins while at the same time improving quality of clinical care, a win for the patient, the hospital, and the payer, almost a total win-win, except for the fact that two stakeholders are absent from this equation: the clinicians and individual cost centers involved in providing non-emergent weekend ERCP service. For this paradigm shift to work, it must be a win for all: the patient, the hospital, the payer, the clinical caregivers, and ERCPproviding cost centers/departments. For this to happen, the benefiting hospital will need to share, adequately and equitably, the cost savings they incur through weekend ERCPrelated reduced LOS with the clinical teams and cost centers providing weekend ERCP service. Effecting twenty-first century clinical care paradigms requires twenty-first century solutions in hospital economics. That just might take what makes sense—and make it happen.

This study shows that non-emergent ERCP performed during the weekend significantlyreduces length of hospital stay (LOS), thereby reducing hospital costs and hospital charges, while increasing hospital margins without negatively impacting clinical outcome

- Reduced LOS decreases patient, payer, and hospital costs; increases hospital access; and may increase patient satisfaction

- Weekend ERCP may thus benefit patient, payer, and hospital

Nevertheless, impact to the caregivers, departments, and cost centers actually involved in providing nonemergent weekend ERCP service must be considered

- Additional weekend work hours of clinicians involved in providing non-emergent weekend ERCP may not be compensated, or adequately compensated, whether in hourly, salaried, or productivity compensated models, particularly if weekend ERCP work is frequent or less efficient than weekday ERCP work

- Non-financial impacts of elective weekend ERCP work are variable and difficult to quantify; disrupting weekends may not be consi dered by some caregivers to be adequately compensated with an equivalent weekday of compensation time

- Compensation for weekend work effort and time may also vary in adequacy for hourly-compensated caregivers

- Negative impacts to caregiver satisfaction can lead to increased staff turnover, increased recruitment and training costs, an d inadequate staffing

Successful and viable non-emergent weekend ERCP service will require hospitals to share financial benefits of weekend ERCP with ERCP-providing cost centers and clinical staff. Adequate clerical and clinical support for caregivers on weekend ERCP teams, along with adequate compensation for time, effort, and lifestyle disruption, so that patients, hospitals, payers, weekend caregivers, and ERCP-providing cost centers alike all benefit from non-emergent weekend ERCP service, will facilitate the ability to provide for non-emergent weekend ERCP service as a durable care model 


\section{Compliance with Ethical Standards}

Conflict of interest The author declares that he has no conflict of interest

\section{References}

1. Abu Dayyeh BK. ERCP: expert consult. Mayo Clin Proc. 2013;88:e111.

2. Adam V, Bhat M, Martel M, et al. Comparison costs of ERCP and MRCP in patients with suspected biliary obstruction based on a randomized trial. Value Health. 2015;18:767-773.

3. Tabibian JH, Yang JD, Baron TH, Kane SV, Enders FB, Gostout CJ. Weekend admission for acute cholangitis does not adversely impact clinical or endoscopic outcomes. Dig Dis Sci. 2016;61:53-61.

4. Inamdar S, Sejpal D, Ullah M, Trindade A. Weekend vs. weekday admissions for cholangitis requiring an ERCP: comparison of outcomes in a national cohort. Am J Gastroenterol. 2016;111:405-410.

5. Hakuta R, Hamada T, Nakai Y, et al. No association of timing of endoscopic biliary drainage with clinical outcomes in patients with non-severe acute cholangitis. Dig Dis Sci. 2018;63:1937-1945.

6. Porter ME. What is value in health care? $N$ Engl J Med. 2010;363:2477-2481.
7. Mathew PJ, Jehan F, Kulvatunyou N, et al. The burden of excess length of stay in trauma patients. Am J Surg. 2018;216:881-885.

8. Lagoe RJ, Johnson PE, Murphy MP. Inpatient hospital complications and lengths of stay: a short report. BMC Res Notes. 2011:4:135.

9. Tokunaga J, Imanaka Y. Influence of length of stay on patient satisfaction with hospital care in Japan. J Qual Health Care. 2002; 14:493-502.

10. Borghans I, Kleefstra SM, Kool RB, Westert GP. Is the length of stay in hospital correlated with patient satisfaction? Int J Qual Health Care. 2012;24:443-451.

11. Hakim S, Aneese AM, Edhi A, Shams C, Purohit T, Cannon M, Cappell MS. A statistically significant reduction in length of stay and hospital costs with equivalent quality of care metrics for ERCPs performed during the weekend versus postponed to weekdays: a six-year study of $533 \mathrm{ERCPs}$ at four teaching hospitals. Dig Dis Sci. (Epub ahead of print). https://doi.org/10.1007/ s10620-020-06066-z.

12. Parikh ND, Issaka R, Lapin B, Komanduri S, Martin JA, Keswani $\mathrm{RN}$. Inpatient weekend ERCP is associated with a reduction in patient length of stay. Gastroenterology. 2014;109:465-470.

Publisher's Note Springer Nature remains neutral with regard to jurisdictional claims in published maps and institutional affiliations. 